\title{
La Calidad del Sueño en los Profesionales Sanitarios de dos Áreas de Salud de Castilla-La Mancha
}

\author{
Yolanda Díaz-Campo García-Carpinteroa , Cristina Díaz-Campo García-Carpinterob, \\ Carmen Puerma Castilloc, M $^{a}$ Nieves Viedma Lloredad ${ }^{d}$, Alberto Aulet Ruize, \\ Eloisa Lázaro Merino ${ }^{\dagger}$, Olga $M^{a}$ Fernández Rodríguez ${ }^{9}$
}

a Enfermera. Hospital General de Tomelloso (Ciudad Real).

${ }^{\mathrm{b}}$ Enfermera. EAP Madridejos (Gerencia de Atención Primaria de Alcázar de San Juan).

\section{${ }^{\mathrm{c}}$ Fisioterapeuta. EAP} Buenavista (Gerencia de atención Primaria de Toledo).

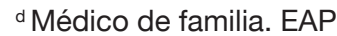
Quintanar de la Orden (Gerencia de Atención Primaria de Alcázar de San Juan).

e Médico de familia. EAP Villafranca de los Caballeros (Gerencia de Atención Primaria de Alcázar de San Juan).

${ }^{\mathrm{f}}$ Médico de familia. Coordinadora de Equipos. Gerencia de AP de Alcázar de San Juan.

${ }^{9}$ Médico de Familia. Técnico de Salud de la Unidad Docente de MFyC de Toledo.

Correspondencia: Yolanda Díaz-Campo García-Carpintero. C/ San Roque, 27, 13250Daimiel (Ciudad-Real). Telf.: 639548543 / 926850655 , e-mail: yulhi@lycos.es

Recibido el 6 de abril de 2008.

Aceptado para su publicación el 28 de mayo de 2008.

\section{RESUMEN}

Objetivos. Conocer las características del sueño en profesionales de atención primaria y su relación con la atención continuada y con la actividad asistencial.

Diseño. Estudio multicéntrico, descriptivo y transversal mediante cuestionario anónimo autocumplimentado.

Emplazamiento. Áreas de Toledo y Alcázar de San Juan.

Participantes. Médicos de familia y profesionales de enfermería.

Mediciones principales. Variables sociodemográficas, de la actividad asistencial y de las características del sueño.

Resultados. Contestaron 281 profesionales, con una edad media de 41,27 años (DE 8,76). La proporción de hombres fue del $37,7 \%$, de médicos el $37,9 \%$ y de los que realizaban guardias el $72,2 \%$. Refirieron trastornos del sueño el 31,7\% (IC95\%: 26,65-38,46), sin diferencias significativas respecto a la realización de guardias, al sexo $(31,6 \%$ hombres vs $31,2 \%$ mujeres) ni a la profesión $(29,7 \%$ médicos vs $32,7 \%$ enfermeros). Para un $72,5 \%$ estos trastornos influían en su actividad habitual y para el $55,6 \%$ en su conducta. Entre los profesionales que realizaban guardias, un $18,8 \%$ tomaba estimulantes durante ellas (sin diferencias por sexo ni profesión) y el 5,5\% relajantes, más los médicos que enfermería $\left(10,7 \%\right.$ vs $\left.1,7 \% ; \chi^{2} 7,66 ; \mathrm{p}<0,01\right)$. El $47,7 \%$ refirió haber cometido errores postguardia, más los médicos $\left(65,1 \%\right.$ vs $\left.34,8 \% ; \chi^{2} 17,7, p<0,001\right)$ y los varones $\left(57,8 \%\right.$ vs $\left.39,4 \% ; \chi^{2} 6,63, p<0,05\right)$. Un $29,6 \%$ había sufrido algún accidente postguardia, siendo mayor en enfermería $\left(21,2 \%\right.$ vs $35 \% ; \chi^{2}$ $4,57 ; p<0,05)$. Para un $76,1 \%$ fue peor el trato a los pacientes postguardia.

Conclusiones. La prevalencia de trastornos del sueño encontrada es similar a la de la población general. Las guardias no parecen asociarse a dichos trastornos, pero sí podrían relacionarse con una peor calidad asistencial y un aumento de los riesgos.

Palabras clave. Trastornos del Sueño, Empleos en Salud, Atención Primaria de Salud.

\section{ABSTRACT}

Sleep quality in healthcare professionals in two health areas in Castilla-La Mancha

Objectives. To determine the characteristics of sleep patterns of primary health care professionals and their relation to continous medical attention and health care.

Design. This is a multicenter, descriptive, cross-sectional study, consisting of a questionnaire completed by the participants.

Setting. Toledo and Alcazar de San Juan health areas.

Participants. General practitioners (GPs) and nurses.

Main measurement. Sociodemographic, health care and characteristic sleep factors.

Results. 281 professionals answered the questionnaire. Average age: 41.27 years (SD 8.76). $37.7 \%$ were men; $37.9 \%$ GPs; $72.2 \%$ were on duty. $31.7 \%$ reported that they have sleep disorders (Cl 95\% $26,65-38,46)$ irrespective of shift work, sex $(31.6 \%$ men vs $31.2 \%$ women) or occupation $(29.7 \%$ GPs vs $32.7 \%$ nurses). For $72.5 \%$ these sleep disorders influenced their daily activity and for $55.6 \%$ it influenced their behaviour. Of the professionals who were on duty, $18.8 \%$ took stimulants while doing shiftwork (there was no difference in sex or occupation) and $5.5 \%$ took sleeping pills, more GPs than nurses $\left(10.7 \%\right.$ vs $\left.1.7 \% ; \chi^{2} 7.66 ; p<0.01\right)$. A total of $47.7 \%$ said that they have made errors when they come off duty, more GPs $\left(65.1 \%\right.$ vs $\left.34.8 \% ; \chi^{2} 17.7, p<0.001\right)$ and men $(57.8 \%$ vs $39.4 \%$ $\left.; \chi^{2} 6.63, \mathrm{p}<0.05\right)$. A total of $29.6 \%$ had had an accident after a night shift. This was more frequent among nurses $\left(21.2 \%\right.$ vs $\left.35 \% ; \chi^{2} 4.57 ; p<0.05\right) .76 .1 \%$ admitted having a worse behaviour towards patients after having been on a shift.

Conclusions. The prevalence of sleep disorders is similar to those of the general population. Shiftwork does not seem to increase these disorders, although they could be associated with a poorer quality of medical care and an increase of risk factors.

Key words. Sleep Disorders, Health Occupations, Primary Health Care.

De este trabajo fue presentada una comunicación en el XXVII Congreso de la SEMFYC, ValladolidSalamanca del 7 al 10 de noviembre de 2007.

El trabajo fue presentado en la X edición de los Premios de Investigación del Área de Toledo, convocados por la Gerencia de Atención Primaria de Toledo. Toledo, 13 de diciembre de 2007. 


\section{INTRODUCCIÓN}

El sueño es una necesidad fisiológica vital para el correcto funcionamiento del organismo. Dormir mal tiene consecuencias adversas para la salud en diferentes niveles: el cansancio, los accidentes por sueño, el absentismo laboral, problemas familiares, sociales, e incluso patologías como la depresión, la úlcera gástrica y el burn-out se asocian habitualmente a la patología del sueño.

Estas consecuencias adversas en la salud, tanto física como psíquica, de la mala calidad del sueño vienen avaladas por numerosos estudios. La privación del $50 \%$ del sueño durante 6 días produce una disminución de la tolerancia a la glucosa, disminución de la tirotropina y aumento excesivo de las concentraciones de cortisol al atardecer, así como de la actividad simpática. Durante la práctica clínica diaria, la privación de sueño causa somnolencia y disminución del rendimiento psicomotor entre los profesionales. La vinculación entre los conceptos "calidad de vida" y "calidad del sueño" parece clara ${ }^{1}$.

La mayor parte de la actividad circadiana ocurre al final de la tarde o principio de la noche, dejando las horas nocturnas dedicadas al descanso. Si se mantiene un tipo de trabajo a turnos durante mucho tiempo o se trabaja exclusivamente en horario nocturno, se va destruyendo el sincronismo ente el reloj interno del cuerpo y el entorno. El asincronismo originado puede causar la aparición de trastornos que propician enfermedades, incluso cambios neurohumorales ligados al sistema inmunológico. Así mismo, padecen alteraciones del ciclo sueño/vigilia con repercusiones sobre el eje hipotálamo-hipófiso-córtico-suprarrenal, a semejanza de aquéllos que realizan un vuelo transoceánico y sufren el fenómeno de jet-lag².
En el Shift Worker Sleep Disorder existe un incremento del riesgo de comorbilidad asociado al trabajo por turnos y, aunque de momento está infrarreconocido e infratratado, conlleva consecuencias sociales, económicas, médicas y de calidad de vida ${ }^{3}$.

El trastorno circadiano por turnicidad indica un patrón de sueño desestructurado ${ }^{4}$, un fenómeno estudiado y reconocido en el medio hospitalario, pero "desconocido" o no valorado en Atención Primaria (AP), en la que la turnicidad no es el patrón de trabajo más frecuente, ya que la jornada laboral suele ser continuada en el caso de hacer guardias. En AP los cambios frecuentes en el horario de sueño se relacionan con dos factores principales: la atención continuada, o guardia, y el hecho de no descansar inmediatamente después de la misma, esperando, mientras se trabaja, a que termine otra jornada laboral completa para poder hacerlo.

Existen pocos estudios sobre la calidad del sueño, los factores que se relacionan con ella y su posible repercusión en los trabajadores sanitarios que realizan atención continuada, a los que no se les puede considerar trabajadores a turnos. Este es el objetivo que nos planteamos en el presente trabajo de investigación, conocer las características del sueño en los profesionales de AP y su relación con la realización de atención continuada y su actividad asistencial.

\section{MATERIAL Y MÉTODO}

Se realizó un estudio multicéntrico, descriptivo y transversal, entre los meses de febrero a junio de 2007. La población de estudio la conformaron todos los médicos de familia y profesionales de enfermería de AP de las Áreas Sanitarias de Toledo y Alcázar de San Juan.

\begin{tabular}{lcc}
\hline & $\begin{array}{c}\text { Realiza al menos } \\
\text { 1 guardia al mes } \\
n=203\end{array}$ & $\begin{array}{c}\text { No realiza } \\
\text { guardias } \\
\mathrm{n}=78\end{array}$ \\
\hline Horario regular de sueño & $70,1 \%$ & $94,1 \%$ \\
Dificultad para conciliar el sueño 1 ó más días a la semana & $54,7 \%$ & $44,9 \%$ \\
Despertares nocturnos 1 ó más días a la semana & $73,2 \%$ & $70 \%$ \\
El sueño es reparador & $42,3 \%$ & $48 \%$ \\
Despertar antes de la hora habitual 1 ó más días a la semana & $45,5 \%$ & $36 \%$ \\
Excesiva somnolencia diurna 1 ó más días a la semana & $55,5 \%$ & $52 \%$ \\
Ronca habitualmente & $14,2 \%$ & $6,4 \%$ \\
Sufre episodios de apnea del sueño habitualmente & $2,1 \%$ & $0 \%$ \\
Sufre pesadillas 1 ó más días a la semana & $41,5 \%$ & $29,4 \%$ \\
Toma ayudas para dormir & $11,2 \%$ & $11,9 \%$ \\
Cree tener problemas de sueño & $34 \%$ & $20,9 \%$ \\
\hline
\end{tabular}

Tabla 1. Características del sueño según realización o no de atención continuada. ${ }^{*}$ ó 7 días a la semana. 


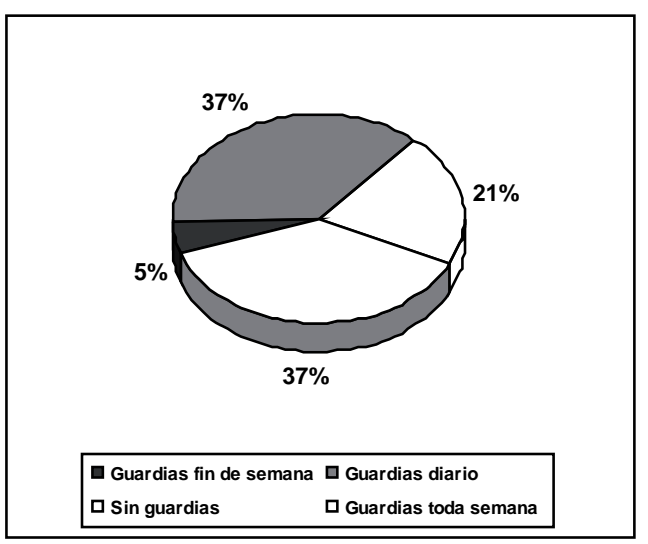

Figura 1. Distribución de los profesionales en función del tipo de guardias que realizan.

Los datos se obtuvieron a través de un cuestionario diseñado "ad hoc" por el propio equipo investigador para ser autocumplimentado. Dicho cuestionario se remitió por correo interno para su autocumplimentación y posterior reenvío a la Unidad Docente de Medicina de Familia y Comunitaria de Toledo. La confidencialidad y anonimato de los profesionales que participaron quedó asegurada en todo momento.

El cuestionario incluía preguntas sobre diferentes variables socio-demográficas: edad, sexo, profesión (médico ó enfermero), tipo de contrato (titular, interino, PEAC, sustituto); sobre la actividad asistencial: número de guardias al mes, distribución temporal de las guardias, consumo de relajantes/estimulantes durante las guardias, repercusión de las guardias en su trabajo y de las características del sueño. Para valorar este último parámetro se utilizaron varias preguntas del cuestionario Oviedo del sueño (COS), instrumento de ayuda diagnóstica para trastornos del sueño según criterios internacionales (CIE 10 y DSM IV), que consta de 11 ítems: dos de ellos interrogan sobre satisfacción con el sueño y el resto sobre patrón de sueño nocturno y diurno, fenómenos anómalos durante el sueño y utilización de soportes para dormir. Junto al COS, se intercalaron preguntas sobre diferentes características del sueño tomadas de la hoja de problemas para los profesionales de enfermería del Programa de Consulta para AP en Castilla La Mancha.

Previamente al inicio del estudio se realizó un pilotaje del cuestionario con 30 voluntarios como método de detección de problemas y corrección de posibles errores de comprensión.

Los datos se recogieron en una base creada a tal efecto y se analizaron mediante el paquete estadístico SPSS 12.0 para Windows, utilizando los parámetros habituales (media, desviación estándar y chi-cuadrado).

\section{RESULTADOS}

Participaron en el estudio 281 profesionales, lo que supone una tasa de respuesta del $39,8 \%$. La edad media de los profesionales que respondieron fue de 41,27 años (DE 8,7). El 37,7\% eran hombres. El 62,1\% eran enfermeros.

Del total de participantes, el $56,1 \%$ eran titulares de la plaza en la que realizan su actividad asistencial, el $19,6 \%$ interinos, el $19,2 \%$ PEAC y el $4,1 \%$ sustitutos. Realizaba atención continuada un $72,2 \%$ de los profesionales, siendo su distribución la que muestra la figura 1.

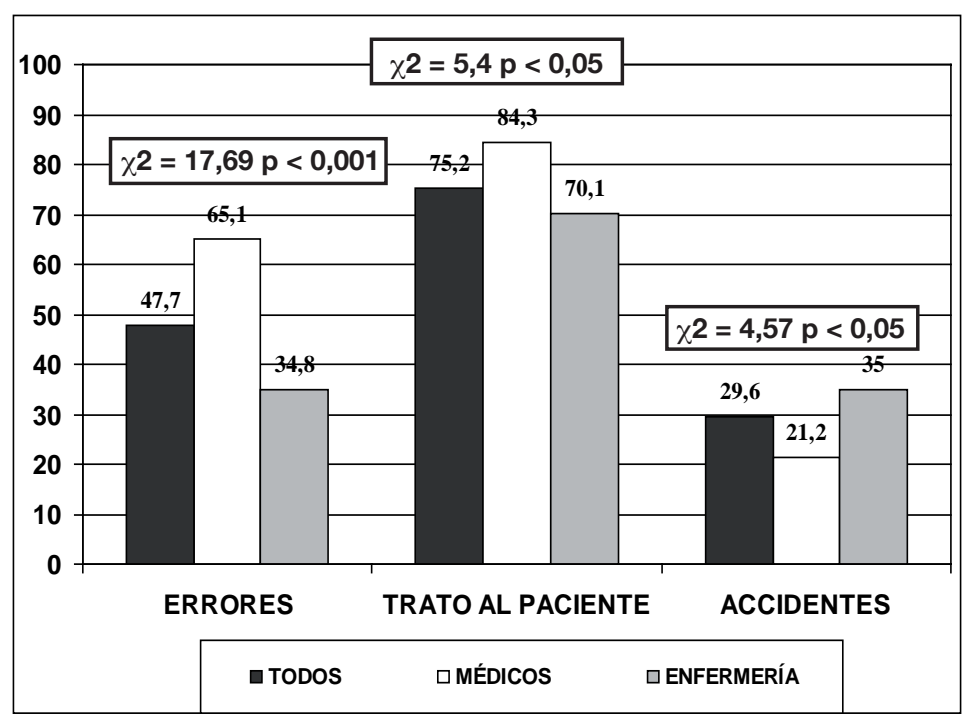

Figura 2. Repercusiones del cansancio postguardia en la atención sanitaria por categoría profesional. 


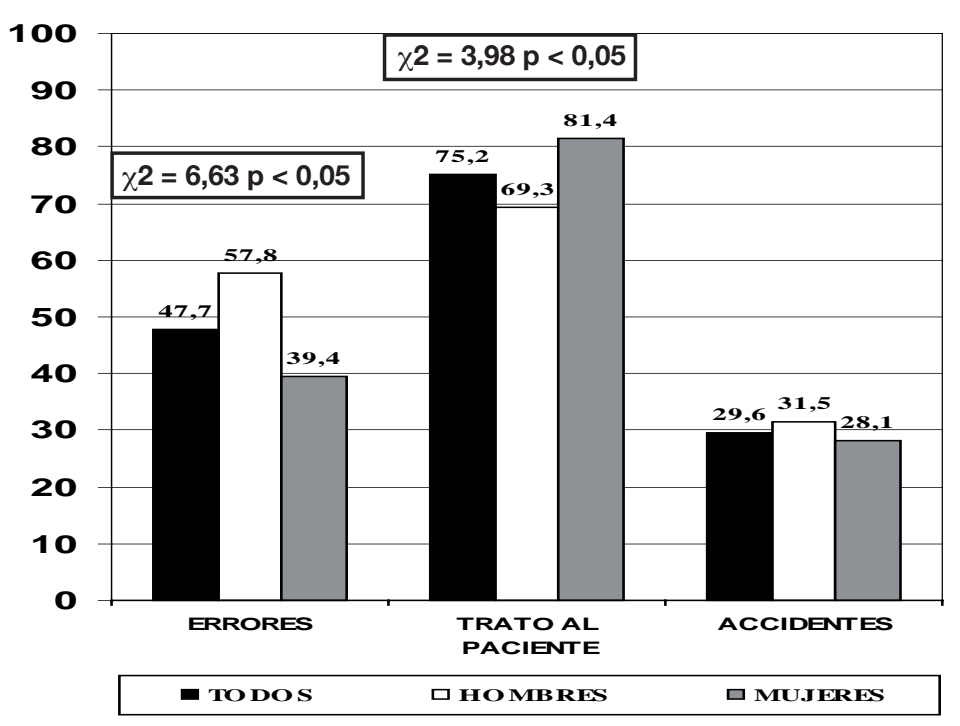

Figura 3. Repercusiones del cansancio postguardia en la atención sanitaria por sexo.

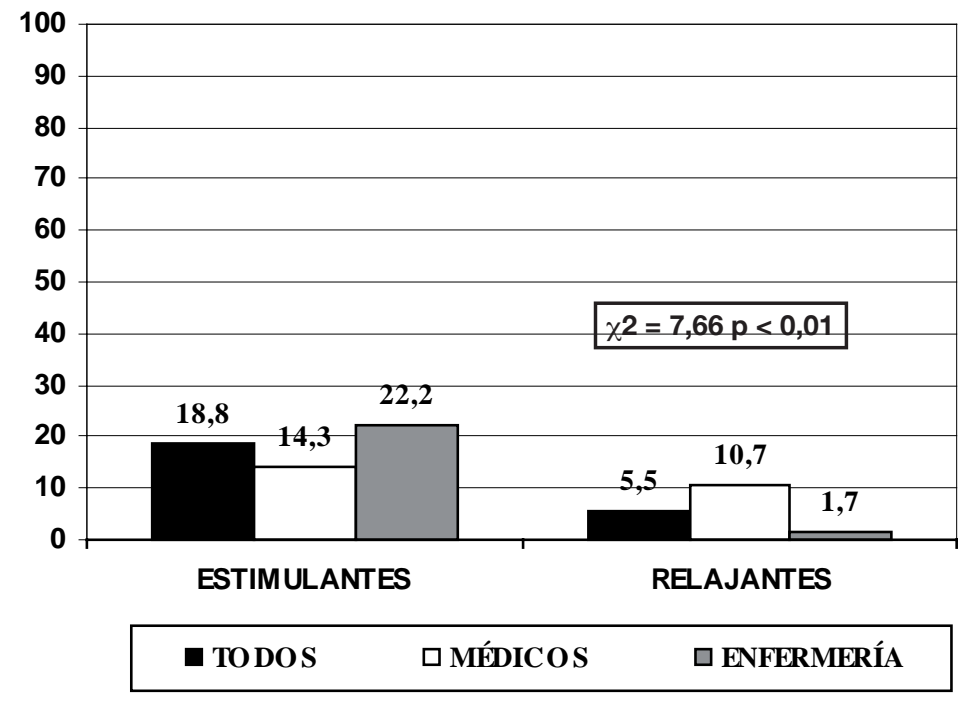

Figura 4. Consumo de estimulantes/relajantes durante la realización de guardias.

El número medio de guardias fue de 1,76 (DE 0,91) en fin de semana y de 3,64 (DE 2,31) en días laborables. La edad media de los profesionales que realizaban guardias fue de 40,7 años (DE 8,92), sin encontrarse diferencias respecto a los que no las realizaban $(41,9$ años; DE 7,7$)$.

Un 63,6\% de los encuestados se mostraba satisfecho con su sueño. Las características del sueño de los profesionales encuestados, distinguiendo entre aquéllos que realizan guardias y los que no, se muestran en la tabla 1.

El 92,3\% del total de la población refería tener una salud buena o normal. Consideraba padecer trastornos del sueño el 31,7\% (IC 95\%: 26.65-38.46) del total de la muestra, sin encontrarse diferencias significativas respecto a la realización o no de guardias, ni al sexo ( $31,6 \%$ hombres vs $31,2 \%$ mujeres) ni a la profesión (29,7\% médicos vs $32,7 \%$ enfermeros).

El 37,8\% de los que tenían trastornos del sueño refería que dicho trastorno influía en su actividad y en el 32,9\% de los casos le ocasionaba alteraciones de la conducta (cansancio en el $24,1 \%$ e irritabilidad en el $13,8 \%$ entre otros). Afirmó levantarse cansado el $39,8 \%$ de los que reconocen tener trastornos del sueño. 
Refiere dar un peor trato a los pacientes tras una guardia el $75,2 \%$ de los encuestados que las realizan en la actualidad, reconociendo esto en mayor porcentaje los médicos $\left(84,3 \%\right.$ vs $\left.70,1 \% ; \chi^{2} 5,40 ; p<0.05\right)$ y las mujeres (81,4\% vs 69,3\%; $\left.\chi^{2} 3.98 ; p<0.05\right)$ (figuras 2 y 3).

El 47,7\% de los sanitarios que realizaban guardias refirieró haber cometido algún tipo de error en la jornada posterior a la guardia, siendo este hecho más frecuente entre los médicos que entre los enfermeros $(65,1 \%$ vs $\left.34,8 \% ; \chi^{2} 17,7 ; p<0,001\right)$ y en los varones $(57,8 \%$ vs $\left.39,4 \% ; \chi^{2} 6,63 ; p<0,05\right)$ (Figuras 2 y 3 ).

Un 29,6\% había sufrido algún tipo de accidente tras la realización de una guardia, sucediendo esto más entre los enfermeros que en los médicos (35\% vs 21,2\%; $\chi^{2}$ 4,57; $p<0,05$ ) (figuras 2 y 3).

Entre los profesionales que realizaban guardias, un $18,8 \%$ tomaba estimulantes durante ellas (sin diferencias significativas respecto al sexo ni a la profesión) y el $5,4 \%$ relajantes, tomándolos más los médicos que los enfermeros $\left(10,7 \%\right.$ vs $\left.1,7 \% ; \chi^{2} 7,66 ; p<0,01\right)$.

\section{DISCUSIÓN}

El presente estudio pretende estimar las posibles alteraciones del sueño existentes entre los sanitarios de atención primaria de nuestras áreas de salud, teniendo en cuenta su diferenciación en dos grupos, en función de que realicen o no atención continuada (guardias). Gran parte de los profesionales de ambas áreas realizan dichas guardias, lo que implica la asunción de jornadas continuadas de más de 24 horas de trabajo (consulta ordinaria, atención continuada y nueva consulta tras la guardia). Este hecho podría implicar que hayan contestado más los profesionales sensibilizados con el hecho de hacer guardias y que consideren que ejercen determinada influencia en su vida y actividad profesional.

De la misma forma, hemos encontrado pocos estudios en la bibliografía consultada relacionando los trastornos del sueño con trabajadores de jornadas continuadas tan prolongadas, salvo los referidos a médicos internos residentes (MIR). Además, la mayoría de los trabajos encontrados se refieren a profesionales sanitarios del ámbito hospitalario y que trabajan haciendo turnos.

La prevalencia de trastornos del sueño encontrada por nosotros es similar a la descrita en diferentes trabajos para la población general ${ }^{6}$, pero no debe olvidarse que dicha prevalencia es subjetiva, ya que no se ha utilizado ningún instrumento validado para valorarla, sino la propia opinión de los profesionales encuestados. Sin embargo, esta percepción es superior entre aquéllos que realizan guardias respecto a los profesionales que no las realizan, lo que parece relacionarse con las alteraciones que puedan generar las guardias.

Independientemente de que poco más de un tercio de los encuestados consideren que padecen trastornos del sueño, observamos cómo al preguntarles sobre diferentes cuestiones acerca de "su sueño", parecen existir alteraciones en un porcentaje muy superior, sobre todo, entre aquéllos que realizan atención continuada. De esta forma, destacan los despertares nocturnos, la excesiva somnolencia diurna, la dificultad para la conciliación del sueño y el despertarse antes de la hora habitual, situaciones que se utilizan para medir la aparición de un trastorno del sueño en la población general.

Para la mayoría de los que tienen trastornos de sueño éstos influyen en su actividad habitual, provocan cansancio, irritabilidad y lentitud, sufren cambios frecuentes en su estado de ánimo, se levantan cansados y, en general, no están satisfechos con su sueño. En la práctica clínica somos testigos de cómo la privación de sueño causa somnolencia y disminución del rendimiento psicomotor. Según algunos estudios, es frecuente la impresión de lentitud y falta de claridad tras no haber dormido, pero su demostración de forma incontestable no ha podido realizarse por la dificultad de medir este fenómeno, entre otros motivos por estar muy influido por la subjetividad de los individuos ${ }^{1}$.

Por otro lado, de entre los que realizan guardias, independientemente de si presentan o no trastornos del sueño, las tres cuartas partes refieren que su trato a los pacientes es diferente al habitual en la jornada posterior a una guardia, posiblemente motivados por la falta de sueño y el cansancio asociados. Existen estudios que informan de cómo entre los trabajadores por turnos aparecen insatisfacción laboral, problemas sociales y tensiones con los compañeros ${ }^{7}$. Igualmente, tampoco podemos atribuir totalmente este hecho a la falta de sueño, ya que el propio cansancio generado por una jornada continuada tan prolongada puede influir en la aparición de esta conducta. En este sentido, según el trabajo realizado por Molina Siguero et al sobre desgaste profesional en un área sanitaria de Madrid, la mayoría de los facultativos consideraba que la elevada carga de trabajo deterioraba la calidad de la asistencia prestada y era la principal causa de estrés en el puesto de trabajo ${ }^{8}$.

Casi la mitad de los sanitarios que realizan guardias refiere haber cometido algún error en su actividad asistencial atribuible a la fatiga causada por la guardia y un tercio ha sufrido accidentes (laborales/extralaborales) que atribuyen a la fatiga causada por la guardia. Este porcentaje es similar al apreciado en un estudio nacional realizado entre MIR donde hasta el 57,9\% de los encuestados creía haber cometido algún error grave motivado por la fatiga causada por el trabajo y un 34,7\% había sufrido algún accidente por el mismo motivo $^{9}$. Igualmente, en estudios norteamericanos, el número de horas trabajadas se relacionaba de forma significativa con el riesgo de sufrir accidentes y cometer errores médicos ${ }^{10}$.

Es destacable, igualmente, la toma de estimulantes (más entre los enfermeros) o relajantes (más entre los médicos) durante las guardias, aunque no sea una práctica generalizada. En el estudio realizado sobre los MIR el porcentaje de consumo sistemático de estas 
sustancias alcanzaba al 17,9\% de la población estudiada, superior al de nuestro estudio ${ }^{9}$. La toma de estimulantes probablemente se relacione con la intención de mantener el nivel de alerta requerido, que se puede ver dificultado por el hecho de dormir de forma irregular y con dificultad. Los relajantes se pueden ver más en consonancia con el control del estrés y de la ansiedad que genera la propia actividad de atención continuada. Igualmente encontramos en la bibliografía cómo en el trabajo por turnos se encuentra un incremento del consumo de sustancias estimulantes o depresoras del sistema nervioso central.

A la luz de las opiniones de nuestros profesionales sanitarios sobre sus trastornos del sueño y actividad asistencial, se desprende que pese a que la prevalencia de trastornos del sueño no es mayor que en la población general y a que las guardias no parecen determinar dichos trastornos, sí podría asociarse la realización de guardias con una peor calidad asistencial y un aumento de los riesgos tanto personales como profesionales si se continúa la jornada laboral tras la guardia. La turnicidad trastorna la calidad del sueño y provoca un malestar importante en la actividad social o laboral del individuo, que no se puede atribuir a otros trastornos del sueño, enfermedad o sustancias y cursa con insomnio en las horas de descanso y somnolencia en las horas de vigilia. Pero se podría asumir que la jornada continuada actuase de forma similar, disminuyendo la capacidad mental e influyendo de forma negativa sobre la calidad de vida y suponiendo un peligro para el profesional y para la seguridad clínica del usuario de nuestro sistema de salud.

Es terrible pensar que cada día, en todos los Centros de Salud con atención continuada, al menos dos profesionales que han tenido guardia (médico y enfermero) pueden cometer errores en relación con el cansancio. Al usuario, víctima de una lotería involuntaria, en cualquier momento le puede tocar el premio de una triste equivocación. Error posiblemente evitable con un cambio en la actual estructura de nuestro sistema de atención sanitaria. Consideramos necesario ajustar la jornada laboral a niveles eficaces de actividad entre los trabajadores sanitarios, si queremos que la calidad asistencial consiga la excelencia a la que nos invita el actual Plan de Salud de Castilla-La Mancha.

\section{BIBLIOGRAFÍA}

1. Fernandez Rodriguez MJ, Bautista Castano I, Bello Lujan L, Fernandez Bethencourt L, Sanchez Villegas A, Serra Majen L. Nutritional evaluation of health shift workers from the Canary Islands. Nutr Hosp. 2004; 19(5):286-91.

2. Martínez- Carpio PA, Corominas A. Introducción general a la cronobiología clínica y a la manipulación terapéutica de los ritmos biológicos. Med Clin (Barc). 2004; 123(6):230-235.

3. Santamaría J. Mecanismos y función del sueño: su importancia clínica. Med Clin (Barc). 2003; 120(19):750-755.

4. Manual diagnóstico y estadístico de los trastornos mentales. Texto revisado. Breviario. Criterios diagnósticos. Barcelona: Masson SA; 2002. p. 256.

5. Bobes García J, González G-Portilla MP, Sáiz Martínez PA, Bousoño García M. Trastornos del sueño. En: Vázquez Barquero JL. Psiquiatría en Atención Primaria. Madrid: Aula Médica; 1998. p. 429-442.

6. Schwartz JR, Roth T. Shift Work sleep disorder: burden of illness and approaches to management. Drugs. 2006; 66(18):2357-70.

7. Choobineh A, Rajaeefard A, Neghab M. Problems related to shiftwork for health care workers at Shiraz University of Medical Sciences. East Mediterr Health J. 2006; 12(3-4):3406.

8. Molina Siguero A, García Pérez MA, Alonso González M, Cecilia Cermeño P. Prevalencia de desgaste profesional y psicomorbilidad en médicos de atención primaria de un área sanitaria de Madrid. Aten Primaria. 2003; 31(9):564-74.

9. Menéndez González M, Ryan Murua P, García Salavarri B. Estudio subjetivo de las guardias sobre la salud, calidad de vida y calidad asistencial de los médicos residentes de España. Arch Med. 2007; 1(3). Disponible en: http://www. sempsph.com/sempsph/attachments/036_guardiasobresalud1-1.pdf

10. Baldwin D, Daugherty S, Tsai R, Scotti M. A National Survey of resident's self-reported work hours:thinking beyond speciality. Acad Med. 2003; 78:1154-1163.

11. Tamagawa R, Lobb B, Booth R. Tolerance of shift work. Appl Ergon. 2007; 38(5):635-42. 